\title{
RADIAL $P$-TH MOMENT OF A RANDOM VECTOR
}

\author{
RigAO HE AND GANGSONG LENG
}

Abstract. In this paper, we first introduce a new concept - radial $p$-th moment of a random vector for star body, which is a general form of the standard $p$-th moment. Further we establish some properties of the radial $p$-th moment and give some related applications.

Mathematics subject classification (2010): 94A17, 60D05, 52A40. Gaussians.

Keywords and phrases: Radial $p$-th moment, Renyi entropy, star body, contour body, generalized

\section{REFERENCES}

[1] Z. Artstein And R. A. Vitale, A strong law of large numbers for random compact sets, Ann. Probab. 5 (3) (1975), 879-882.

[2] J. Bourgain And V. D. Milman, New volume ratio properties for convex symmetric bodies in $R^{n}$, Invent. Math. (88) (1987), 319-340.

[3] K. M. BALL, An elementary introduction to modern convex geometry, Flavors of Geometry, ed. by Silvio Levy, Cambridge University Press, New York, (31) (1997), 1-58.

[4] M. H. M. COSTA AND T. M. COVER, On the similarity of the entropy power inequality and the Brunn-Minkowski inequality, IEEE Trans. Inform. Theory. 6 (IT-30) (1984), 837-839.

[5] T. M. Cover, A. Dembo, And J. A. Thomas, Information theoretic inequalities, IEEE Trans. Inform. Theory. (37) (1991), 1501-1518.

[6] T. M. Cover And J. A. Thomas, Elements of information theory, New York: Wiley-Interscience, (1991).

[7] D. Cordero-Erausquin, B. Nazaret, and C. Villani, A mass-transportation approach to sharp Sobolev and Gagliardo-Nirenberg inequalities, Adv. Math. 2 (182) (2004), 307-332.

[8] A. Dembo, Information inequalities and uncertainty principles, Dep. Statist., Stanford Univ., Stanford, CA, Tech. Rep. (1991).

[9] R. J. Gardner, Geometric tomography, Cambridge University Press, Cambridge, (1995).

[10] P. M. Gruber, Convex and discrete geometry, Springer-Verlag, New York, (2007).

[11] O. G. Guleryuz, E. LutwaK, D. YANG, AND G. ZHANG, Information-theoretic inequalities for contoured probability distributions, IEEE Trans. Inform. Theory. 8 (48) (2002), 2377-2383.

[12] R. He AND G. LENG, A strong law of large numbers on the harmonic p-combination, Geom. Dedicata, (154) (2011), 103-116.

[13] R. He AND G. LENG, A probabilistic version of quermassintegrals' Brunn-Minkowski inequality (in Chinese), Sci. Sin. Math, (43) (2013), 941-952.

[14] B. S. KASIN, The widths of certain finite-dimensional sets and classes of smooth functions, Izv. Akad. Nauk SSSR Ser. Mat. 2 (41) (1977), 334-351.

[15] E. LUTwAK, The Brunn-Minkowski-Firey theory II: Affine and Geominimal Surface Areas, Adv. Math. (118) (1996), 244-294.

[16] E. LutwaK, D. YAnG, AND G. ZHAng, A new ellipsoid associated with convex bodies, Duke Math. J. 3 (104) (2000), 375-390.

[17] E. Lutwak, D. YAng, And G. Zhang, $L_{p}$ affine isoperimetric inequalities, J. Differential Geom. 1 (56) (2000), 111-132.

[18] E. Lutwak, D. YAng, And G. Zhang, The Cramer-Rao inequality for star bodies, Duke Math. J. 1 (112) (2002), 59-81. 
[19] E. Lutwak, D. Yang, AND G. Zhang, Moment-entropy inequalities, Ann. Probab. (32) (2004), $757-774$.

[20] E. Lutwak, D. YANG, AND G. Zhang, Cramer-Rao and moment-entropy inequalities for Renyi entropy and generalized Fisher information, IEEE Trans. Inform. Theory. (51) (2005), 473-478.

[21] E. LUTwaK, D. YANG, AND G. ZHANG, Moment-entropy inequalities for a random vector, IEEE Trans. Inform. Theory. (53) (2007), 1603-1607.

[22] E. Lutwak, S. Lv, D. YANG, AND G. Zhang, Extensions of Fisher Information and Stam's Inequality, IEEE Trans. Inform. Theory. (58) (2012), 1319-1327.

[23] E. LuTwAK, S. Lv, D. YANG, AND G. ZHANG, Affine moments of a random vector, IEEE Trans. Inform. Theory. (59) (2013), 5592-5599.

[24] S. Lv, AND X. Lv, Affine Fisher information inequalities, J. Math. Anal. Appl. (371) (2010), 347-354.

[25] R. Schneider, Convex bodies: the Brunn-Minkowski theory, Cambridge University Press, Cambridge, 1993.

[26] R. A. Vitale, On the volume of parallel bodies: a probabilistic derivation of the Steiner formula, Adv. in appl. Probab. (27) (1995), 97-101.

[27] R. A. Vitale, The Wills functional and Gaussian processes, Ann. Probab. (24) (1996), 2172-2178. 